\title{
The Mitochondrial ATP Synthase: Improved Resolution Cryo-EM Model and Insight into the Structure of the Peripheral Stalk Region
}

\author{
John L. Rubinstein \\ Structural Biology and Biochemistry Program, Research Institute \\ The Hospital for Sick Children \\ 555 University Avenue \\ Toronto, Ontario \\ Canada \\ M5G 1 X8
}

The ATP synthase is a large, membrane-bound macromolecular machine. The enzyme uses the energy of the proton motive force across the inner mitochondrial membrane to synthesize ATP, the chemical energy currency of cells. We previously published a model of the intact ATP synthase structure at $32 \AA[1]$. We are developing improved image analysis methods to refine the resolution of the model. We have used an objective test of alignment accuracy [2] to assess different alignment strategies. Using this approach we have determined that we are able to improve particle alignments by differentially weighting spatial frequencies in the Fourier transform of particle images prior to alignment (Figure 1). Improving particle alignments allows for a higher-resolution model to be built for a given dataset (Figure 2). Even at lower resolution than is necessary for chemical information to be apparent, docking atomic models of component subunits and subcomplexes into the map gives insight into the arrangement of subunits within the complex. Using this approach, recent crystallographic studies have revealed the structure of most of the peripheral stalk region of the complex.

1. J. L. Rubinstein, J.E. Walker and R. Henderson. EMBO J. 22 (2003) 6182.

2. P. B. Rosenthal and R. Henderson. J. Mol. Biol. 333 (2003) 721. 

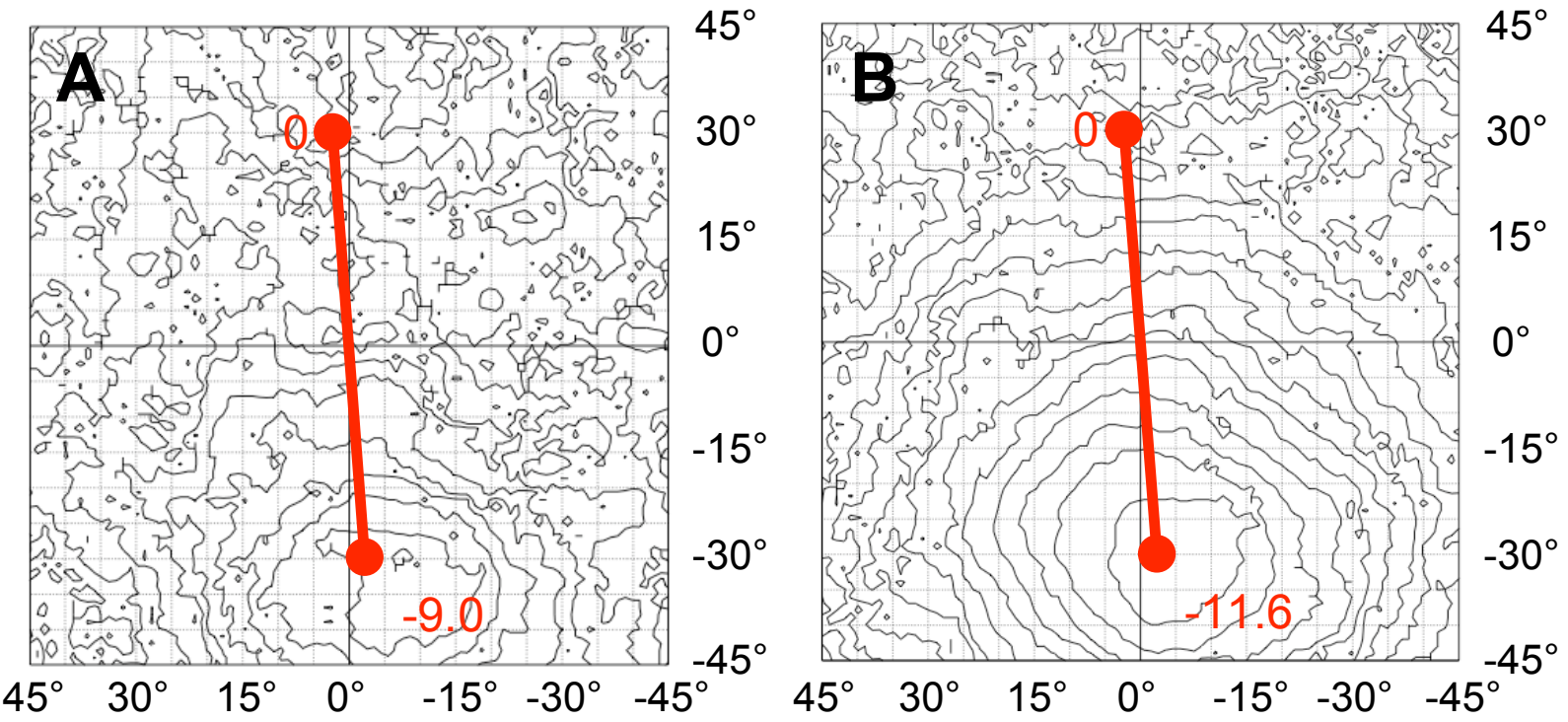

Figure 1. Measurement of the accuracy of particle alignment using the method of Rosenthal and Henderson [2]. Phase residual plots indicate that particle alignment accuracy may be improved by differential weighting of the spatial frequencies in particle images prior to alignment. Parts A and B compare unweighted and weighted particle images, respectively.
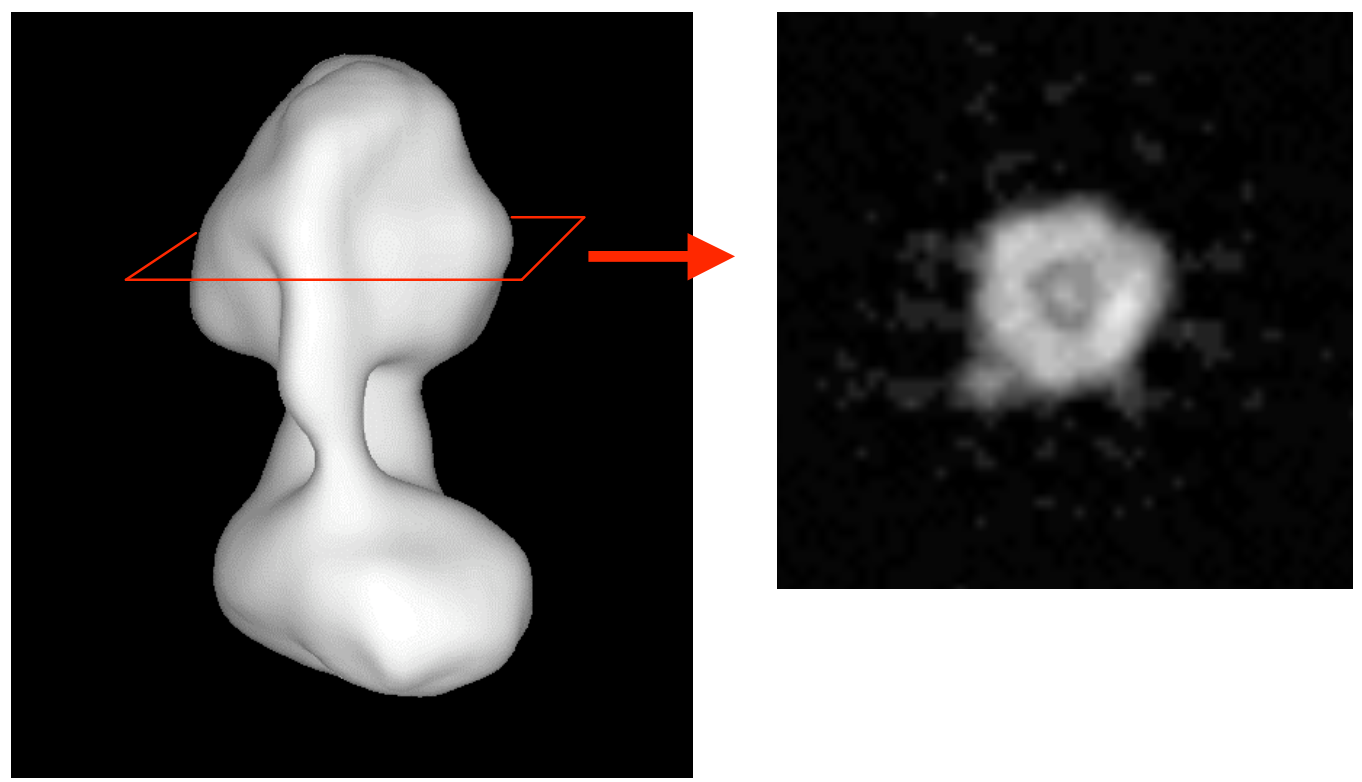

Figure 2. Improved ATP synthase model. More accurate alignments gives rise to a more accurate model of the ATP synthase. 